bedenkt, daß die archäologischen Objekte großenteils Kopien darstellen, die zudem häufig schlecht oder falsch restauriert sind. Selbst die Zuweisung der ursprünglichen Originale an berühmte Bildhauer der Antike ist fragwürdig. In jeder vordergründigen Ähnlichkeit eine «tiefere Affinität» zu suchen, wäre nicht beweisbar. C.Isler-Kerényi stört sich offenbar auch an dem Begriff der personifizierten Wunschvorstellung, der natürlich ein Hilfsbegriff ist, den man aber gebrauchen muß, bis ein besserer gefunden ist.

In der Zwischenzeit erschien der Lexikonartikel «Hygieia» im LIMC, V, I und II, Artemis-Verlag Zürich und München 1990, von Francis Croissant, dessen Ergebnisse sich mit meiner Abhandlung vergleichen lassen.

Hildegard Sobel

D-6509 Undenheim

\title{
Communication de la rédaction
}

Le comité de rédaction de Gesnerus avait prévu de publier à la fin de l'été 1991 le volume contenant les communications présentées au Symposium sur l'histoire de la médecine, de la santé publique et de la biologie en rapport avec la Suisse romande qui s'est tenu à Genève les 5 et 6 octobre 1990 dans le cadre de la réunion annuelle de la Société suisse d'histoire de la médecine et des sciences naturelles. Ce volume devait constituer la livraison 2/3 de l'année en cours. Mais l'ampleur des textes à publier a dépassé les prévisions et retardé la préparation du volume. Celui-ci paraîtra donc au début de 1992 et constituera la livraison $3 / 4$ du volume 48 .

Nous présentons donc ici selon le rythme habituel de parution de notre revue le deuxième cahier de l'année 1991 qui contient comme à l'ordinaire des contributions touchant à des domaines variés de l'histoire de la médecine et des sciences naturelles et nous présentons à nos lectrices et à nos lecteurs nos vœux pour d'heureuses fêtes de fin d'année. 